\title{
ANATOMICALLY BASED ABLATION OF ATRIAL FLUTTER IN AN ACUTE CANINE MODEL OF THE MODIFIED FONTAN OPERATION
}

Mark D. Rodefeld, MD ${ }^{\mathrm{a}}$

Sanjiv K. Gandhi, MD ${ }^{\mathrm{a}}$

Charles B. Huddleston, $\mathrm{MD}^{\mathrm{a}}$

Bryan J. Turken, BS ${ }^{a}$

Richard B. Schuessler, $\mathrm{PhD}^{\mathrm{a}}$

John P. Boineau, MD ${ }^{\text {a }}$

James L. Cox, MD ${ }^{\mathrm{a}}$

Burt I. Bromberg, $\mathrm{MD}^{\mathrm{b}}$
Background: Lateral tunnel total cavopulmonary connection, also called the modified Fontan operation, uses a baffle through the right atrium. We established, in an acute canine model, that atrial flutter after total cavopulmonary connection revolves around a line of conduction block imposed by the free wall lateral tunnel suture line. We hypothesized that a line of conduction block between the free wall total cavopulmonary connection suture line and the tricuspid anulus would interrupt atrial flutter in this model. Objective: Our objective was to determine whether a cryolesion placed between the free wall total cavopulmonary connection suture line and the tricuspid anulus would terminate atrial flutter in an acute canine model. Methods: Seven adult dogs underwent median sternotomy and institution of cardiopulmonary bypass. A suture line was placed through a right atriotomy to simulate total cavopulmonary connection lateral tunnel construction. Form-fitting 253-point biatrial endocardial mapping electrodes were placed via bilateral ventriculotomies. Atrial flutter was induced by atrial burst pacing. A cryothermal lesion was then placed between the free wall total cavopulmonary connection suture line and the tricuspid anulus in the low lateral right atrium (i.e., CRYO 1 procedure), and reinduction of atrial flutter was attempted. If atrial flutter was reinduced, the cryolesion was modified superiorly to include the caudal portion of the atriotomy (i.e., CRYO 2 procedure). Activation sequence maps were generated for sinus rhythms before and after the cryolesions were placed and for induced arrhythmias. Results: In all seven cases, atrial flutter was inducible after suture line placement, before placement of a cryolesion. The reentrant circuit incorporated both caval orifices in five of seven cases and was successfully ablated by the CRYO 1 approach in each case. Atrial flutter was not inducible after placement of the CRYO 2 lesion in the remaining two cases, in which breakthrough of the wave front occurred across the lateral tunnel suture line in the intercaval region. Activation sequence maps of sinus rhythm after placement of the cryolesions demonstrated a conduction block at the site of the lesion. Conclusions: A linear cryothermal lesion placed between the free wall aspect of the total cavopulmonary connection suture line and the tricuspid anulus created a line of conduction block that successfully ablates atrial flutter in the canine model. (J Thorac Cardiovasc Surg 1996;112:898-907)
A rial flutter (AFL), or intraatrial reentrant tachycardia, is a common early and late postoperative complication in patients undergoing the modified

From the Department of Surgery, Division of Cardiothoracic Surgery, and the Department of Pediatrics, Division of Pediatric Cardiology, ${ }^{\mathrm{b}}$ Washington University School of Medicine, St. Louis, Mo.

Supported by National Institutes of Health grants HL32257 and HL33722. Dr. Rodefeld was supported by a National Research Service Award grant (HL08894).

Received for publication Feb. 7, 1996; revisions requested March 11, 1996; revisions received March 25, 1996; accepted for publication March 26, 1996.
Fontan operation, or lateral tunnel total cavopulmonary connection (TCPC). AFL after TCPC has a prevalence ranging from $5 \%$ to $30 \%$ at 5-year follow-up. ${ }^{1-5}$ Reliance on the TCPC for palliation of more complex congenital cardiac malformations has resulted in an increasing number of patients vulner-

Address for reprints: Burt I. Bromberg, MD, Assistant Professor of Pediatrics, Department of Pediatrics, Division of Cardiology, Suite 1S40, St. Louis Children's Hospital, One Children's Place, St. Louis, MO 63110.

Copyright (C) 1996 by Mosby-Year Book, Inc.

$0022-5223 / 96 \$ 5.00+0 \quad \mathbf{1 2 / 1 / 7 3 7 8 5}$ 
able to the hazards of postoperative supraventricular arrhythmias and the antiarrhythmic medications used as therapy. ${ }^{6}$ Although AFL after TCPC is a substantial problem, definitive treatment that would alleviate the need for medical therapy is not yet available.

We previously demonstrated that the TCPC suture line alone, without any hemodynamic alterations, provides an anatomic substrate for AFL in an acute canine model. ${ }^{7}$ The reentrant pathway depends on an isthmus of myocardium between the free wall TCPC suture line and the tricuspid anulus. We hypothesized that creation of a line of conduction block between these two nonconducting boundaries would eliminate AFL after TCPC repair. The objective of this study was to determine whether a cryolesion placed between the free wall TCPC suture line and the tricuspid anulus would terminate AFL in a canine model.

\section{Methods}

Simulated modified Fontan operation and insertion of mapping electrodes. Seven adult mongrel dogs weighing 25 to $30 \mathrm{~kg}$ were anesthetized with intravenous pentobarbital sodium $(30 \mathrm{mg} / \mathrm{kg})$, intubated, and mechanically ventilated using a volume-cycled ventilator. An adequate plane of anesthesia was maintained by intermittent infusion of 1 to $2 \mathrm{mg}$ of pentobarbital sodium. Limb lead electrocardiogram was monitored, and a 20 -gauge arterial pressure catheter was placed in the left femoral artery. Median sternotomy was performed, the azygos vein occluded, and the heart cradled in the pericardium. Bipolar pacing and sensing electrodes were sutured to the right and left atrial appendages, respectively. After administration of heparin $(1 \mathrm{mg} / \mathrm{kg})$, normothermic cardiopulmonary bypass was instituted with a $16 \mathrm{~F}$ arterial cannula in the right femoral artery and with $28 \mathrm{~F}$ bicaval venous cannulas. A right atriotomy was made from the midatrial appendage, anterior to the crista terminalis, to the level of the sinus node artery in the lower right atrial free wall.

A continuous 4-0 polypropylene suture (no baffle) was placed to simulate TCPC lateral tunnel construction (Fig. 1). The suture line started at the limbus of the fossa ovalis and traveled inferiorly between the coronary sinus and the inferior vena caval orifice, around the inferior vena cava, laterally up the crista terminalis, around the superior vena caval orifice, and back to the fossa ovalis. The atriotomy was closed with continuous 4-0 polypropylene suture.

Two form-fitting silicone elastomer (Dow Corning Corp., Midland, MI) atrial endocardial electrode templates containing 253 unipolar electrodes were inserted through bilateral ventriculotomies. These electrodes were designed to fit the complex endocardial contours of the atria (Fig. 2). They were constructed from plaster casts obtained from postmortem formalin-fixed canine atria after the TCPC suture line had been placed. The distance between unipolar electrode points was 6 to $8 \mathrm{~mm}$. After excision of the mitral and tricuspid valve leaflets, the

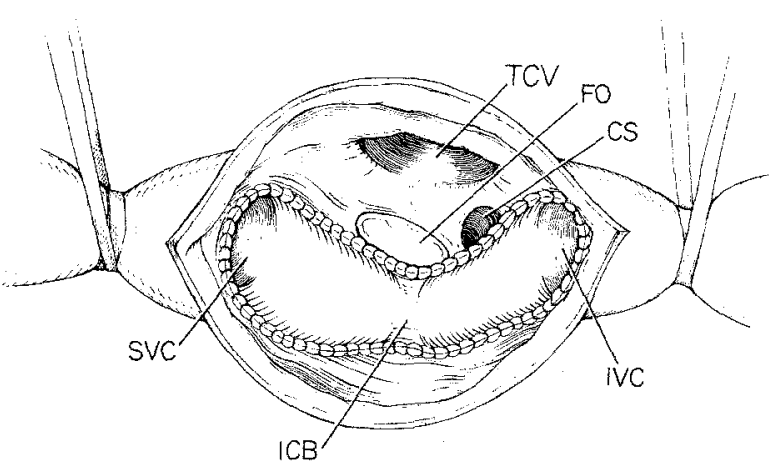

Fig. 1. Surgeon's view of the total cavopulmonary connection lateral tunnel suture line. The suture line starts at the limbus of the fossa ovalis and travels inferiorly between the coronary sinus and the inferior vena caval orifice, around the inferior vena cava, laterally up the crista terminalis, around the superior vena caval orifice, and back to the fossa ovalis. CS, Coronary sinus; $F O$, fossa ovalis; $I C B$, intercaval band; $I V C$, inferior vena cava; $S V C$, superior vena cava; $T C V$, tricuspid valve. (From Rodefeld MD, Bromberg BI, Schuessler RB, Boineau JP, Cox JL, Huddleston CB. Atrial flutter after lateral tunnel construction in the modified Fontan operation: a canine model. J Thorac Cardiovasc Surg 1996;111:514-26.)

electrodes were positioned and anchored to each anulus. Placement was confirmed by palpation through the atrial myocardium. An indifferent electrode was attached to the chest wall for unipolar reference.

All animals received humane care in compliance with the "Principles of Laboratory Animal Care" formulated by the National Society for Medical Research and the "Guide for the Care and Use of Laboratory Animals" prepared by the National Academy of Science and published by the National Institutes of Health (NIH Publication No. 86-23, revised 1985). The study protocol was approved by the Washington University Animal Studies Committee.

Pacing protocol. Atrial burst pacing at cycle lengths ranging from 100 to $300 \mathrm{msec}$ was achieved with a programmable pulse generator (Bloom Inc., Reading, Pa.). Stimulus input was set at twice the pacing threshold. Attempts to induce AFL were made after the lateral tunnel suture line was placed, the atriotomy closed, and the mapping electrodes inserted. Sustained AFL was defined as a stable tachycardia lasting more than 30 seconds that exhibited a fixed atrial cycle length of less than 250 msec. Reproducibility was determined by reinduction of the tachyarrhythmia by means of the same extrastimulus pattern that originally induced it. In cases in which sustained AFL could not be induced, isoproterenol (3 $\mu \mathrm{g} / \mathrm{kg}$ ) was administered and the pacing protocol repeated.

Cryoablation of AFL. After the induction of AFL, an epicardial cryoablative lesion was placed in a linear and 

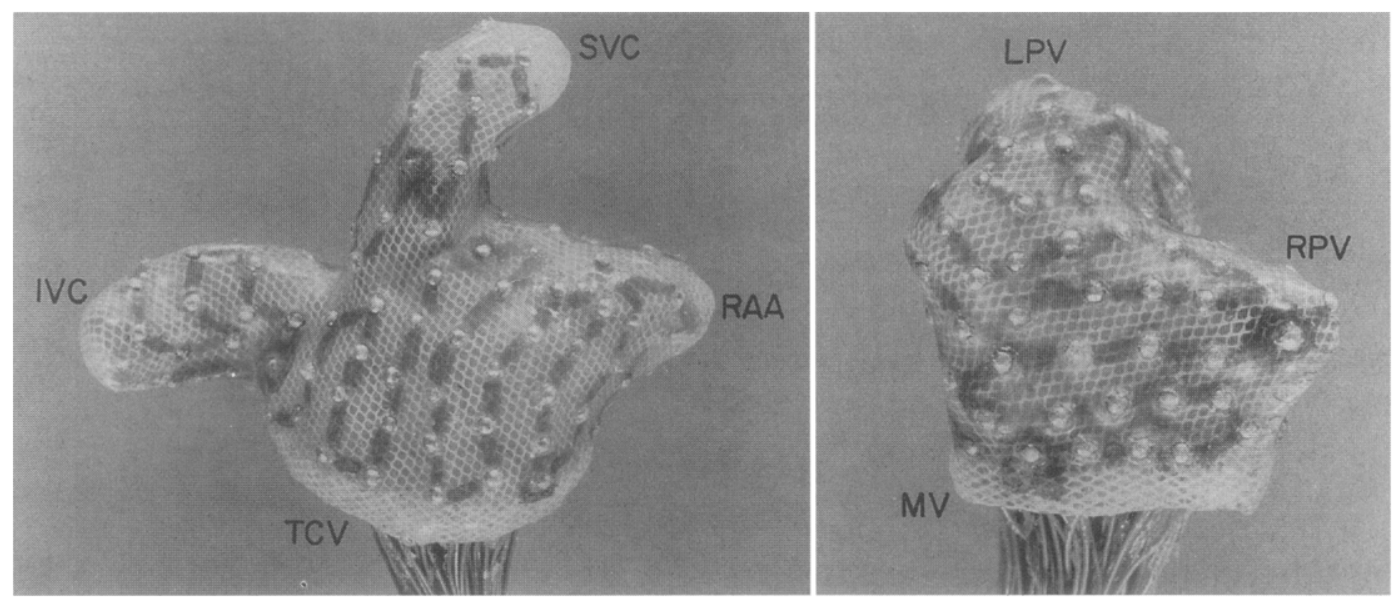

Fig. 2. Right and left atrial endocardial electrode templates containing 253 unipolar points. The electrodes were constructed from plaster casts obtained from postmortem, formalin-fixed canine atria after the total cavopulmonary connection suture line had been placed. $I V C$, Inferior vena cava; $L P V$, left pulmonary veins; $M V$, mitral valve; $R A A$, right atrial appendage; $R P V$, right pulmonary veins; $S V C$, superior vena cava; $T C V$, tricuspid valve.

A

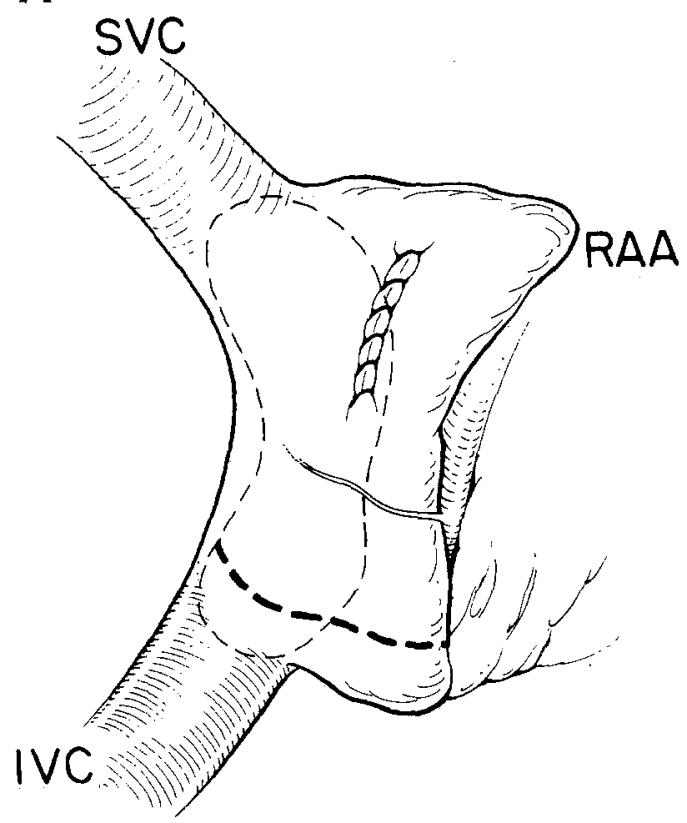

B

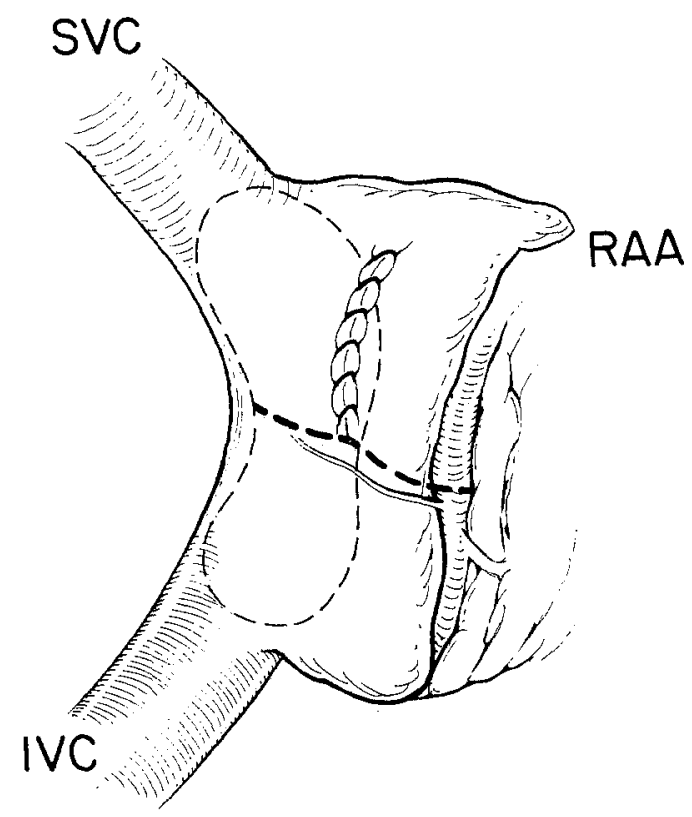

Fig. 3. Epicardially placed cryothermal lesions. The thin dotted lines indicate the position of the intraatrial lateral tunnel suture line. The thick dotted lines represent the cryolesion. The atriotomy is depicted on the free wall. A, In CRYO I, the cryothermal lesion was placed from the free wall segment of the TCPC suture line to the tricuspid anulus, in the low lateral right atrium. B, In CRYO II, the lesion was placed from the free wall segment of the TCPC suture line to the tricuspid anulus, incorporating the inferior edge of the atriotomy. $I V C$, Inferior vena cava; $R A A$, right atrial appendage; $S V C$, superior vena cava.

stepwise fashion in the low lateral right atrium from the free wall lateral tunnel suture line, proximal to the inferior vena cava, to the tricuspid anulus (i.e., CRYO 1 approach; Fig. $3, A$ ). Reinduction of AFL was then attempted, as previously described in the Pacing protocol section. If reinduction of the arrhythmia was successful, the cryolesion was modified superiorly to include the caudal extent of the atriotomy (i.e., CRYO 2 approach; Fig. 3, B). 

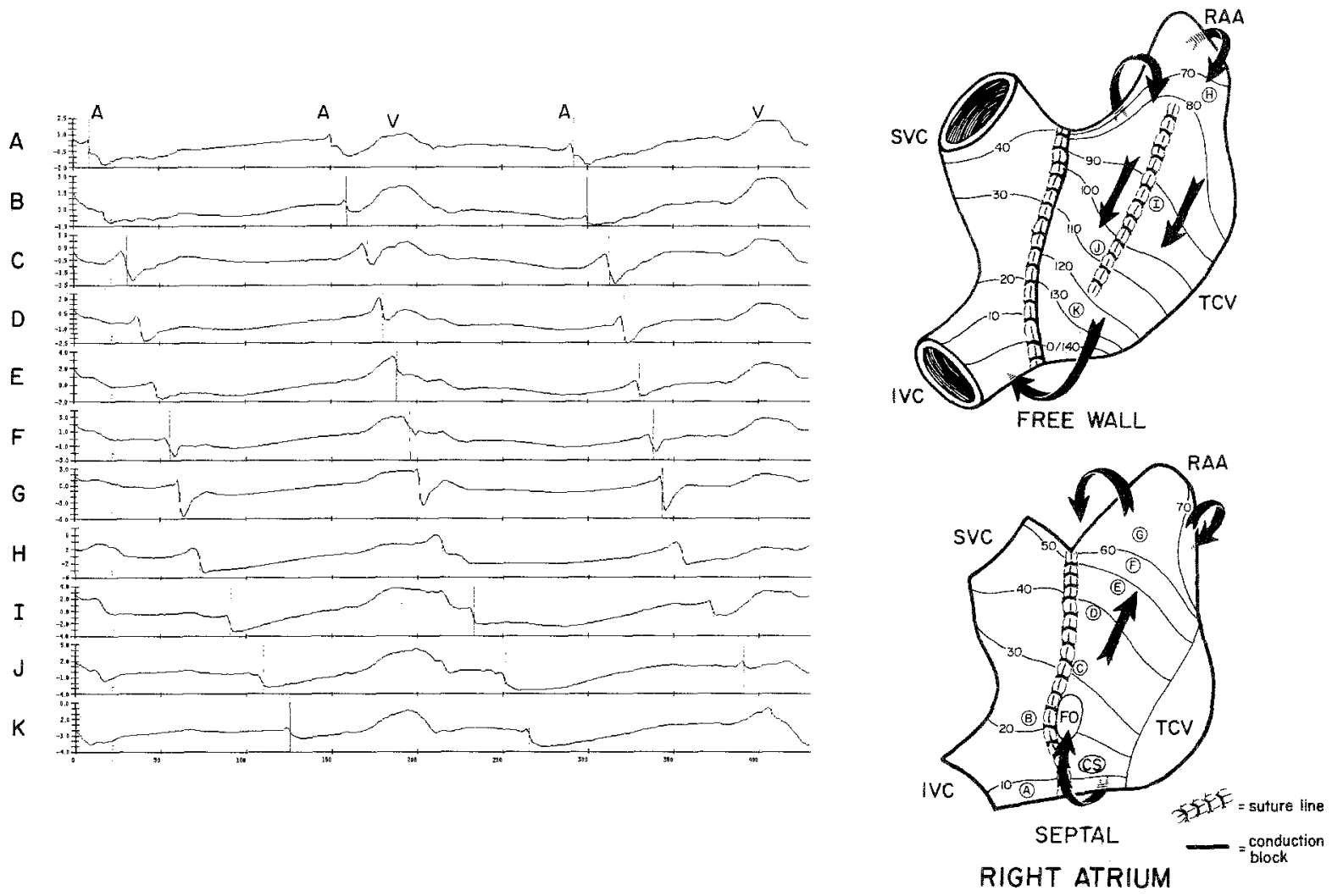

Fig. 4A. Atrial activation during atrial flutter. The free wall of the right atrium is displayed in the upper panel, and the septal surface is shown in the lower panel. The total cavopulmonary connection (TCPC) suture line is depicted on the free wall and septal views. The atriotomy is illustrated on the free wall. Time isochrones demonstrate clockwise rotation around a line of conduction block imposed by the lateral TCPC suture line in continuity with the caval orifices. As the wave front moves rostrally along the septum ( $A$ through $G$ ) onto the free wall surface $(H)$, the suture line serves as a line of conduction block. Conduction along either side of the atriotomy is uniform. After traveling down the free wall ( $I$ through $K$ ), the wave front emerges around the inferior vena cava back onto the septum to complete the reentrant circuit. Electrograms corresponding to sites $A$ through $K$ are also demonstrated. CS, Coronary sinus; $F O$, fossa ovalis; $I V C$, inferior vena cava; $R A A$, right atrial appendage; $S V C$, superior vena cava; $T C V$, tricuspid valve.

Data acquisition and analysis. Atrial activation sequence data were obtained by simultaneously recording 253 unipolar electrograms from the endocardial templates. Data were recorded during sinus rhythm and during any sustained arrhythmia by means of a 256 channel computerized data acquisition and analysis system based on a VaxStation II/GPX graphics workstation (Digital Equipment Corp., Maynard, Mass.) connected to two 128-channel PDP 11/23+-based data acquisition subsystems (LKC Technologies, Inc., Gaithersburg, Md.). ${ }^{7}$ The system is run with software (i.e., GLAS) developed in house. Computer-generated activation sequence maps were reconstructed from all recordings. Data processing and display were performed on a Silicon Graphics Iris 4D/320GTX high-performance graphic workstation (Silicon Graphics Inc., Mountain View, Calif.). Activation sequence maps were displayed as realtime images on a three-dimensional model of the atrium. ${ }^{8}$

\section{Results}

Induction of AFL and activation sequence mapping. In all seven dogs, sustained AFL was reproducibly inducible after lateral tunnel suture line placement, with a mean cycle length of $179 \pm 22$ msec. Isoproterenol administration was required in two cases. As has been previously described, ${ }^{7}$ the free wall segment of the TCPC suture line resulted in a line of conduction block that was evident during sinus rhythm and AFL. During AFL, this line of block created an isthmus between it and the tricuspid anulus through which the reentrant circuit propagated (Fig. 4A). The AFL pathway was confined to the right atrium in all cases. The reentrant circuit most commonly revolved around 

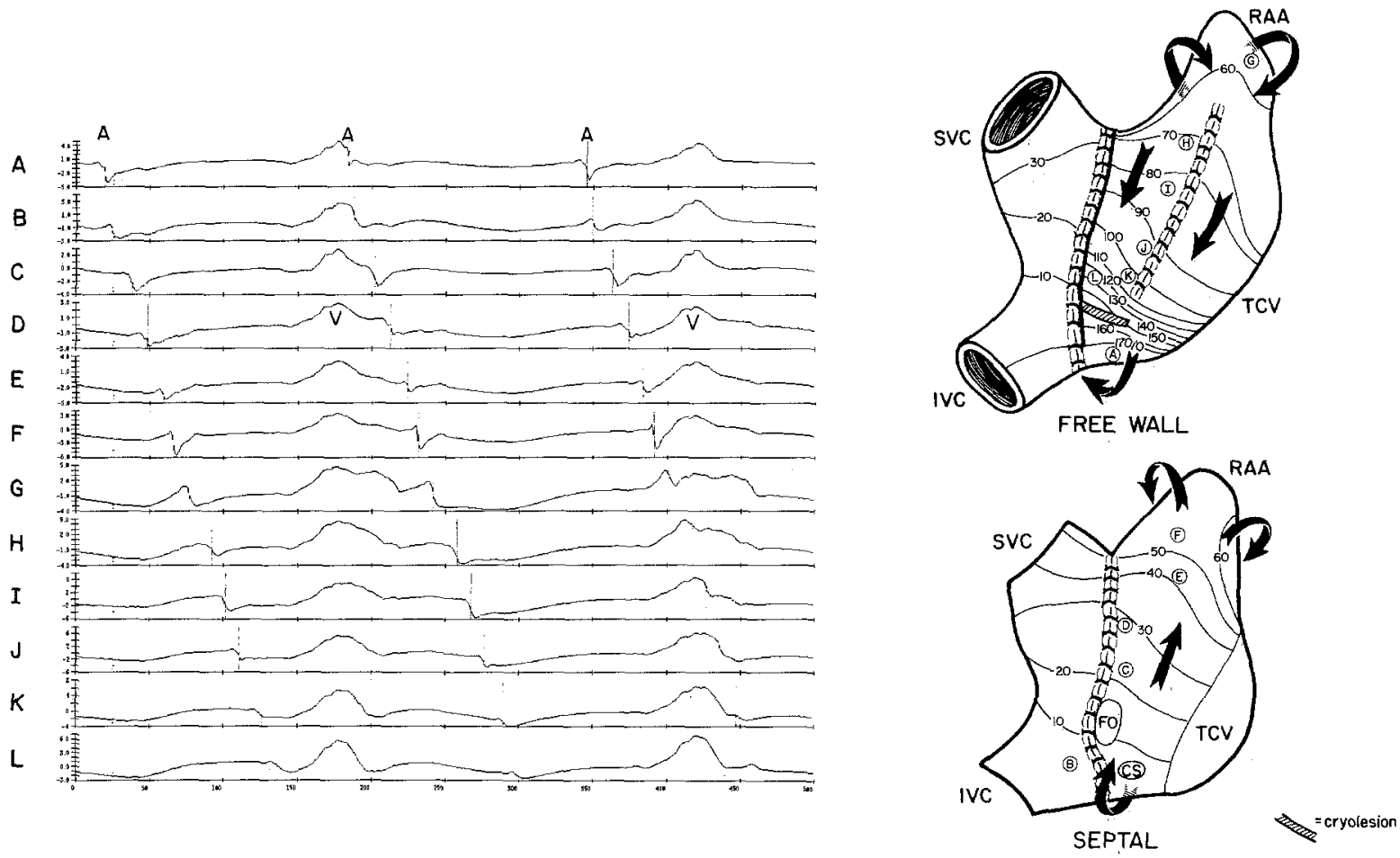

RIGHT ATRIUM

Fig. 4, Cont'd. B, During placement of the cryolesion from the free wall segment of the TCPC suture line to the tricuspid anulus, the atrial flutter cycle length increases from 140 to $170 \mathrm{msec}$, secondary to profound slowing of conduction in the low lateral right atrium in the vicinity of the lesion, as evidenced by the "crowding" of time isochrones in this region. Electrograms corresponding to sites A through $\mathrm{L}$ accompany the activation sequence maps. For abbreviations see Fig. 4A.

both caval orifices. However, in two instances, breakthrough occurred across the suture line between the superior and inferior venae cavae (Fig. $5 \mathrm{~A})$.

Cryosurgical ablation of AFL. Placement of the CRYO 1 lesion resulted in the termination of sustained AFL in five of seven cases. Reinduction of AFL was not possible in these five cases, even after the administration of isoproterenol. Gradual lengthening of the reentrant circuit typically occurred during application of the cryoablative lesion (Fig. 4B). In the two cases in which activation sequence maps revealed breakthrough of the propagating wave front across the free wall suture line between the superior and inferior venae cavae, CRYO 1 failed to terminate the reentrant circuit. The wave front emerged superior to this line of block (Fig. 5B). Superior extension of the cryolesion to include the inferior aspect of the atriotomy (i.e., CRYO 2) successfully terminated the tachyarrhythmia in these instances. In all seven experiments, a complete linear ablative lesion between the free wall suture line and the tricuspid anulus was required to terminate AFL; ablation was never successful with only a partial cryolesion.

Activation sequence maps during sinus rhythm, after placement of the cryolesions, demonstrated a line of conduction block between the lateral tunnel suture line and the tricuspid anulus, in addition to the conduction block imposed by the free wall suture line (Fig. 6). Before the cryolesion was made, the free wall was activated by dual wave fronts, one traveling rostrally after breaking through the suture line inferiorly and one moving caudally after crossing onto the free wall from the septum. The cryolesion blocked the inferior impulse, resulting in rostrocaudal activation of the free wall. 


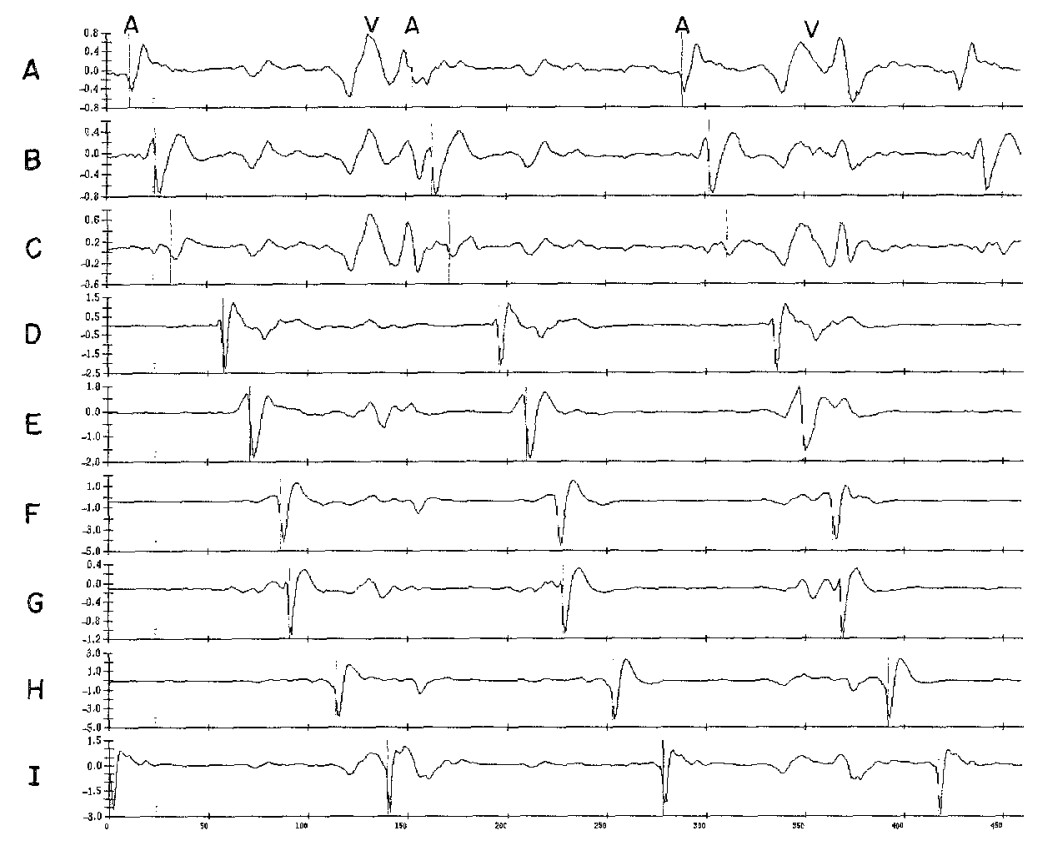

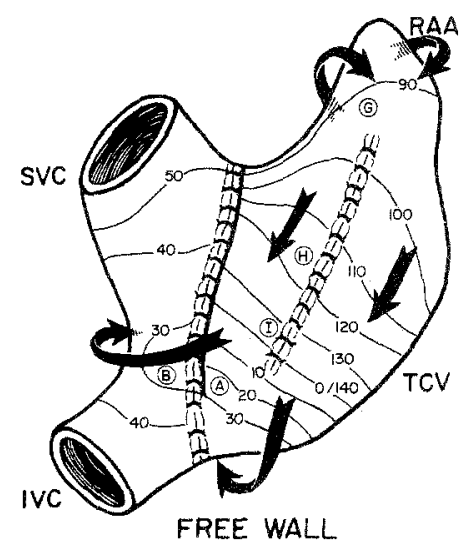

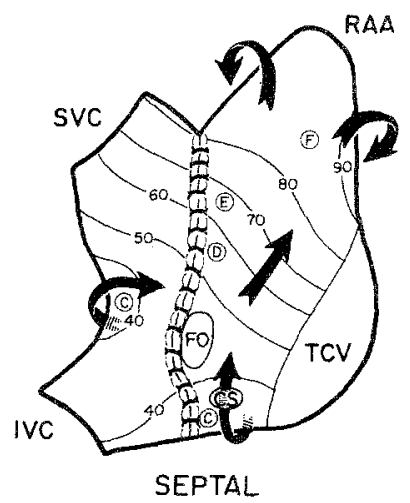

RIGHT ATRIUM

Fig. 5A. Atrial activation during atrial flutter in which breakthrough across the suture line occurs. Time isochrones demonstrate a clockwise reentrant loop. Along the caudal portion of the suture line, between the inferior and superior vena cavae, breakthrough of the advancing impulse occurs $(A$ and $B$ ). The wave front travels around both sides of the inferior vena cava onto the septal surface $(C)$. Propagation up the septum is rapid and uniform $(D$ through $F$ ). The wave front traverses the right atrial appendage back onto the free wall ( $H$ and $I$ ) to complete the reentrant circuit. Electrograms corresponding to sites A through I accompany the activation sequence maps. $C S$, Coronary sinus; $F O$, fossa ovalis; $I V C$, inferior vena cava; $R A A$, right atrial appendage; $S V C$, superior vena cava; $T C V$, tricuspid valve.

\section{Discussion}

Termination of reentry by interruption of a critical isthmus. Interrupting conduction at any point within a reentrant circuit should terminate the tachycardia. After TCPC, two fixed anatomic barriers in the low lateral right atrium, the free wall TCPC suture line and the tricuspid anulus, serve as nonconducting boundaries for an isthmus of tissue that provides an essential substrate for AFL. A cryolesion interrupting this isthmus was successful in terminating AFL, confirming the reentrant mechanism of the arrhythmia and the critical nature of the isthmus. The lesion did not create a substrate for novel reentrant pathways, further establishing its potential utility as an ablative approach.
The importance of a critical isthmus in the inferior right atrium with respect to the genesis of AFL has been established in numerous experimental and clinical investigations. The isthmus of myocardium between the inferior vena cava and tricuspid anulus has been demonstrated to be integral to the reentrant circuits in the canine crush, ${ }^{9}$ enlargement, ${ }^{10}$ and sterile pericarditis ${ }^{11}$ models of AFL. A similar corridor has been documented in the common form of spontaneous human AFL. ${ }^{12}$

Spinelli and colleagues, ${ }^{13}$ who used the AFL model of Frame and coworkers, ${ }^{14}$ illustrated that the site of AFL termination by several different antiarrhythmic medications was located in the same isthmus between the inferior vena cava and tricuspid 

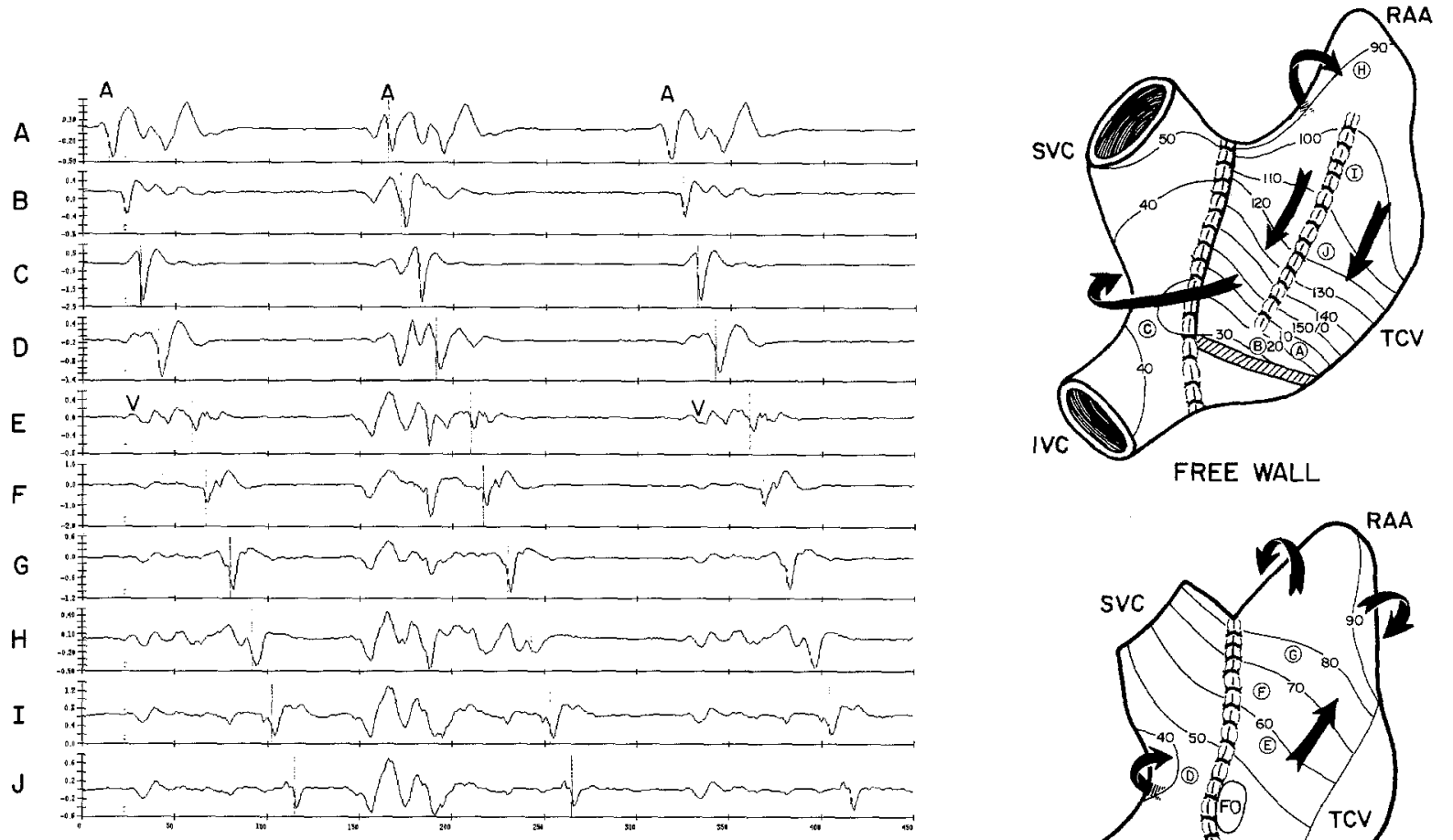

FREE WALL

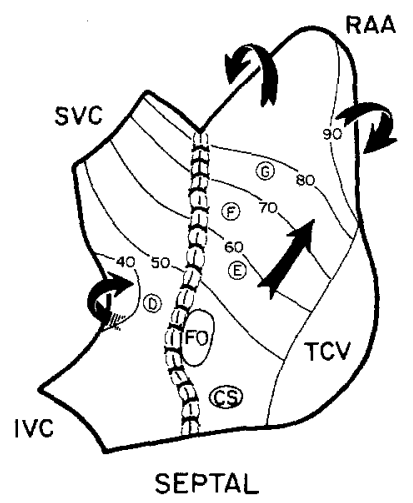

RIGHT ATRIUM

Fig. 5, Cont'd. B, After a cryolesion is placed between the free wall segment of the suture line and the tricuspid anulus in the low lateral right atrium (i.e., CRYO I procedure), the loop continues superior to the line of block imposed by the lesion ( $A$ through $C$ ). Placement of CRYO II, which incorporated the distal aspect of the atriotomy, succeeded in terminating atrial flutter in this case. The accompanying electrograms correspond to sites $A$ through J. For abbreviations see Fig. 5A.

anulus. Termination of the tachyarrhythmia was preceded by a marked increase in the cycle length of AFL, similar to the results produced in the current study. That conduction block at the initiation of drug-induced termination of AFL preferentially localizes to this area of focal conduction in the low lateral right atrium is a consistent feature of several different animal models. ${ }^{10,15}$

The inferior right atrium has also been targeted as a site for the surgical ablation of AFL. Tabuchi and colleagues ${ }^{16}$ who also used Frame's model of AFL, successfully terminated reentry by ablating the isthmus between the inferior vena cava and tricuspid anulus mechanically and with transvenous radiofrequency energy lesions. Isber and associates ${ }^{17}$ applied an epicardial cryosurgical lesion to a similar zone in the sterile pericarditis model of AFL. Analogous to our results, as the inferior margin of the line of conduction block moved closer to the atrioventricular ring, the decreasing width of the resultant isthmus of tissue progressively lengthened the reentrant circuit. Termination occurred when the isthmus was completely interrupted. Klein and colleagues ${ }^{18}$ mapped the isthmus between the inferior vena cava and tricuspid anulus intraoperatively and successfully ablated it with a linear surgical cryolesion.

In addition to successfully interrupting the reentrant pathway, the cryolesion used at this site has several beneficial characteristics. Because the distance between nonconductive barriers in the low lateral right atrium is relatively short, a minimal ablative lesion is required. This region is accessible from the epicardial surface, permitting expeditious application of the cryolesion. This location also avoids nearby important conductive tissue such as 

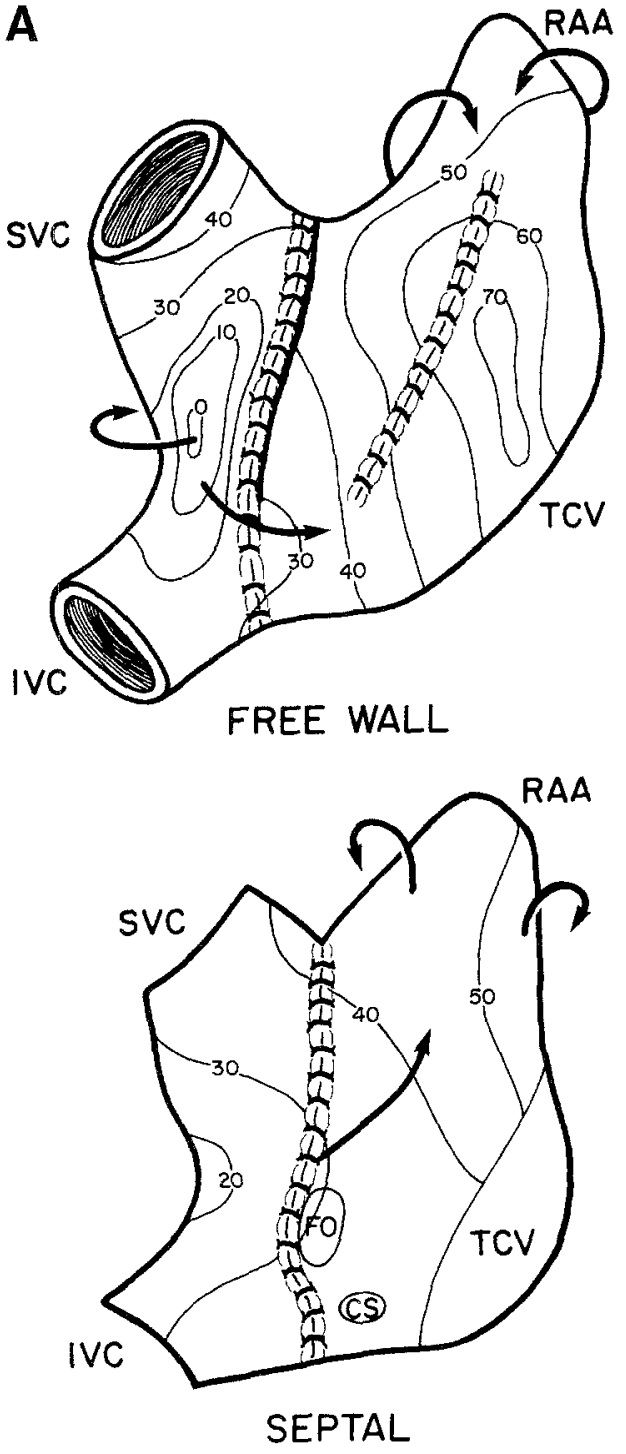

RIGHT ATRIUM

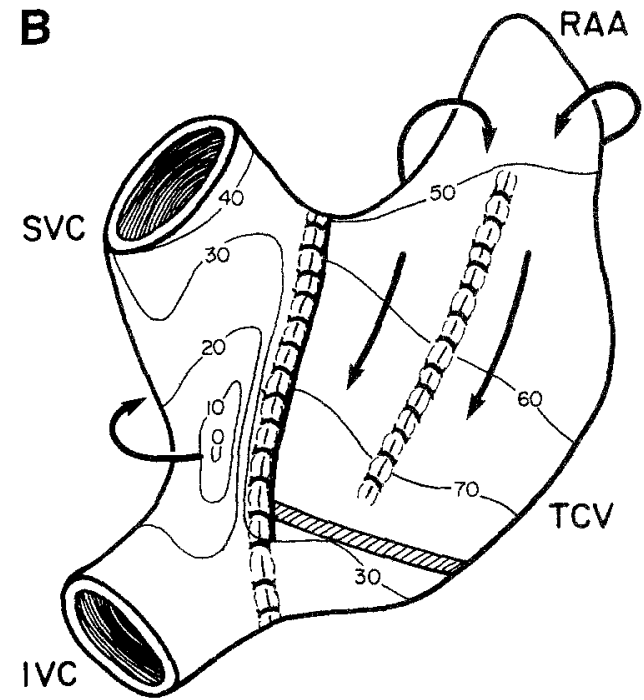

FREE WALL

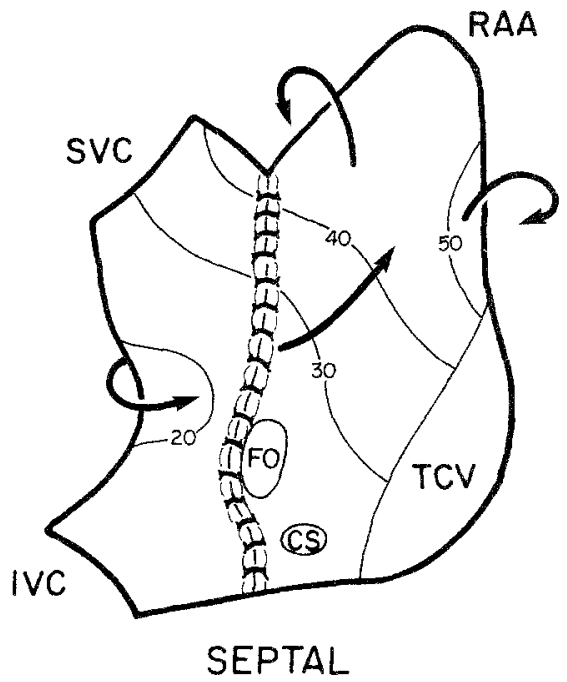

RIGHT ATRIUM

Fig. 6. Atrial activation during sinus rhythm. A, On the free wall, the wave front propagates from the sinus node and encounters a zone of block that corresponds to the free wall portion of the total cavopulmonary connection suture line. One area on the caudal end of the suture line permits breakthrough of the wave front. The impulse from the sinus node reaches the septal side by traveling around either side of the superior vena cava. It then moves rapidly and uniformly along the septum and crosses back onto the free wall. The free wall is activated caudally and rostrally. B, After application of CRYO I, the wave front, after breaking through the caudal aspect of the suture line, encounters a line of conduction block imposed by the cryolesion. The free wall is consequently activated in a rostrocaudal direction after the impulse crosses onto it after traveling up the septum. $C S$, Coronary sinus; $F O$, fossa ovalis; $I V C$, inferior vena cava; $R A A$, right atrial appendage; $S V C$, superior vena cava; $T C V$, tricuspid valve.

the sinoatrial and atrioventricular nodes and the crista terminalis. Another advantage is that the tissue in this region is relatively simple anatomically.
Clinical implications. AFL continues to be a significant problem after the modified Fontan repair, despite the putative hemodynamic advantages of this operation. ${ }^{19}$ The electrophysiologic ablation 
of AFL after TCPC has not been previously reported. Catheter ablation for other forms of postoperative AFL has had varied results. Most series report that either recurrence or overt failure is prevalent. ${ }^{20,21}$ However, many investigators have used radiofrequency point lesions at the exit sites of slow conduction. Anatomically guided approaches have yielded more success. ${ }^{22,}{ }^{23}$ In our model, on the right atrial free wall, the transmurally placed suture line causes tissue injury sufficient to produce a complete line of conduction block in most instances. A distinct area of slow conduction is not present in the AFL circuit, and it is the anatomic site of an isthmus between nonconductive barriers that is critical for the successful interruption of reentry.

Clinical studies are often restricted by the $a b-$ sence of reliable atrial activation sequence mapping. The relative paucity of simultaneously recorded points of activation makes it difficult to delineate areas of conduction disturbance and rationally propose the length and orientation of effective linear ablative lesions. This limitation is magnified after TCPC for which, with the use of standard venous access, atrial recording sites are restricted to those within the lateral tunnel. Our canine model suggests that the crucial portion of the circuit is located exterior to the baffle. Consequently, transvenous ablation of AFL after TCPC requires access to the pulmonary venous side of the baffle. This increases the technical difficulty of the procedure, because it mandates advancing a catheter retrogradely through the aortic valve, where it can then be prolapsed across the systemic atrioventricular valve. For patients with intractable AFL, a surgically applied ablative lesion may be a feasible option.

Limitations of the study. Caution must be exercised in extrapolating data from this study to patients with AFL after TCPC. The animals in our model had normal cardiac anatomy. It is difficult to speculate how the anatomic and physiologic influences of complex congenital cardiac malformations, such as tricuspid atresia and hypoplastic left heart syndrome, and the hemodynamic alterations after TCPC may have influenced the results. Although the cryolesion produced a line of conduction block in the short term, establishing permanent transmural myocardial damage requires a long-term study design. Moreover, the potential of the cryolesion as a prophylactic modification to prevent AFL after TCPC was not evaluated.

\section{Conclusion}

A linear cryothermal lesion placed between the free wall aspect of the TCPC suture line and the tricuspid anulus creates a line of conduction block that successfully ablates AFL for the short term in a canine model. Further investigations are warranted to determine the long-term utility of this technique.

We are grateful to the technical staff of the Washington University Cardiothoracic Research Laboratory. We would also like to thank the Surgical Illustrations Department for their assistance in preparation of the figures.

\section{REFERENCES}

1. Driscoll DJ, Offord KP, Feldt RH, Schaff HV, Puga FJ, Danielson GK. Five to fifteen year follow-up after Fontan operation. Circulation 1992;85:469-96.

2. Balaji S, Gewillig M, Bull C, de Leval MR, Deanfield JE. Arrhythmias after the Fontan procedure: comparison of total cavopulmonary connection and atriopulmonary connection. Circulation 1991;84(Suppl):III162-7.

3. Cecchin F, Johnsrude CL, Perry JC, Friedman RA. Effect of age and surgical technique on symptomatic arrhythmias after the Fontan procedure. Am J Cardiol 1195;76:386-91.

4. Pearl JM, Laks H, Stein DG, Drinkwater DC, George BL, Williams RG. Total cavopulmonary anastomosis versus conventional modified Fontan procedure. Ann Thorac Surg 1991;52:189-96.

5. Paul T, Zeimer G, Luhmer I, Hecker H, Kallfelz HC. Early and late atrial dysrhythmias after modified Fontan operation: implications of preoperative hemodynamics and type of operation (atriopulmonary vs total cavopulmonary connection). Z Kardiol 1993;82:368-75.

6. Balaji S, Johnson TB, Sade RM, Case CL, Gillette PC. Management of atrial flutter after the Fontan procedure. J Am Coll Cardiol 1994;23:1209-15.

7. Rodefeld MD, Bromberg BI, Schuessler RB, Boineau JP, Cox JL, Huddleston CB. Atrial flutter after lateral tunnel construction in the modified Fontan operation: a canine model. J Thorac Cardiovasc Surg 1996;111:514-26.

8. Branham BH, Bi X, Cox JL. A system for accurate interactive 3-D display of cardiac electrical activity: Institute of Electrical and Electronic Engineers. 1992;20:335-8.

9. Rosenblueth A, Garcia Ramos J. Studies on flutter and fibrillation: II. The influence of artificial obstacles on experimental auricular flutter. Am Heart J 1947;33:677-84.

10. Schoels W, Kuebler E, Yang H, Gough WB, El-Sherif N. A unified functional/anatomic substrate for circus movement atrial flutter: activation and refractory patterns in the canine right atrial enlargement model. J Am Coll Cardiol 1993;21: 73-84.

11. Schoels W, Gough WB, Restivo M, El-Sherif N. Circus movement atrial flutter in the canine sterile pericarditis model: activation patterns during initiation, termination, and sustained reentry in vivo. Circ Res 1990;67:35-50.

12. Cosío FG, López-Gil M, Goicolea A, Arribas F, Barroso JL. Radiofrequency ablation of the inferior vena cava-tricuspid valve isthmus in common atrial flutter. Am J Cardiol 1993; 71:705-9.

13. Spinelli W, Hoffmann BF. Mechanisms of termination of 
reentrant atrial arrhythmias by class I and class III antiarrhythmic agents. Circ Res 1989;65:1565-79.

14. Frame LH, Page RL, Hoffman BF. Atrial reentry around an anatomic barrier with a partially refractory excitable gap. Circ Res 1986;58:495-511.

15. Yamashita $T$, Inoue $H$, Nozaki A, Kuo T-t, Usui M, Ajiki K, et al. Role of anisotropy in determining the selective action of antiarrhythmics in atrial flutter in the dog. Cardiovasc Res 1992;26:244-9.

16. Tabuchi T, Okumura K, Matsunaga T, Tsunoda R, Jougasaki $\mathrm{M}$, Yasue $\mathrm{H}$. Linear ablation of the isthmus between the inferior vena cava and tricuspid annulus for the treatment of atrial flutter: a study in the canine atrial flutter model. Circulation 1995;92:1312-9.

17. Isber N, Restivo M, Gough WB, Yang H, El-Sherif N. Circus movement atrial flutter in the canine sterile pericarditis model. cryothermal termination from the epicardial site of the slow zone of the reentrant circuit. Circulation 1993;87:1649-60.

18. Klein GJ, Guiraudon GM, Sharma AD, Milstein S. Demonstration of macroreentry and feasibility of operative therapy in the common type of atrial flutter. Am J Cardiol 1993;57: $587-91$.

19. de Leval MR, Kilner P, Gewillig M, Bull C. Total cavopulmonary connection: a logical alternative to atriopulmonary connection for complex Fontan operations. J Thorac Cardiovasc Surg 1988;96:682-95.

20. Triedman JK, Saul JP, Weindling SN, Walsh EP. Radiofrequency ablation of intra-atrial reentrant tachycardia after surgical palliation of congenital heart disease. Circulation 1995;91:707-14.

21. Van Hare GF, Lesh MD, Stanger P. Radiofrequency catheter ablation of supraventricular arrhythmias in patients with congenital heart disease: results and technical considerations. J Am Coll Cardiol 1993;22:883-90.

22. Cosío FG, Goicolea A, López-Gil M, Arribas F. Catheter ablation of atrial flutter circuits. PACE 1993;16:637-42.

23. Kirkorian G, Monacada E, Chevalier P, Canu G, Claudel JP, Bellon $\mathrm{C}$, et al. Radiofrequency ablation of atrial flutter: efficacy of an anatomically guided approach. Circulation 1994;90:2804-14. 